\title{
Management Information and Model of GSMP Network Open Interface
}

\author{
YoungWook Cha ${ }^{1}$, TaeHyun Kwon ${ }^{1}$, ChoonHee Kim² ${ }^{2}$ and JunKyun Choi $^{3}$ \\ ${ }^{1}$ Andong National University, Korea \\ \{ywcha, taehyun\}@andong.ac.kr \\ ${ }^{2}$ Daegu Cyber University, Korea \\ chkim@dcu.ac.kr \\ ${ }^{3}$ Information and Communications University, Korea \\ jkchoi@icu.ac.kr
}

\begin{abstract}
General switch management protocol (GSMP) is a network open interface between a label switch and a controller, and it provides connection, configuration, event, performance management and synchronization. There will be two considerations for network management services in GSMP interfaces. The first one is what kind of management information base will be used for network management services in GSMP interface. The second consideration is where network management functions for network management services will be located in GSMP interface. We guided the usage of management information base for each network management service. We proposed the network management model, in which management functions are distributed in a controller and a label switch according to network management services.
\end{abstract}

\section{Introduction}

Network technology is moving form monoliths to component-based network elements. In the environment of component-based network elements, the open interface allows the technologies of forwarding and control to evolve independently from each other [1-2]. General switch management protocol (GSMP) provides a network open interface that can be used to separate the data forwarder from the routing and other control plane protocols [3]. GSMP has been considered to be an open interface in several organizations such as a multi-service switching forum, IEEE programmable interface network, and automatic switched optical network in ITU-T [1].

Figure 1 shows the GSMP interface between a controller and a label switch. GSMP protocol is asymmetric, the controller being the master and the switch being the slave. This means that the controller makes decisions to establish connection and routing, and that the switch merely responds to the commands from the controller. GSMP allows a controller to establish connections across the label switch, manage switch ports, request configuration information, reserve switch resources, and gather statistics. It also allows the label switch to inform the controller of asynchronous events such as a link going down [3]. 


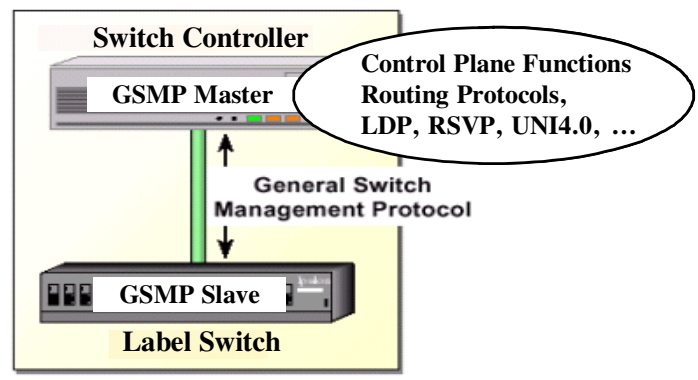

Fig. 1. GSMP Interface

Traditionally, network management (NM) services are classified into five categories: configuration, performance, fault, accounting, and security management services. Network management functions for these services can be located in either a controller or a label switch. There had been no considerations for NM services in GSMP interface before the draft document [4].

There are two considerations for network management services in GSMP interfaces. The first one is what kind of management information base (MIB) will be used for network management services in GSMP interface. GSMP protocol MIB of RFC 3295 [5] is just a protocol MIB, which cannot support traditional network management services. We guided the usage of MIBs for each network management service in the GSMP interface. The second consideration is where network management functions for network management services will be located in the GSMP interface. We proposed the network management model, in which management functions are distributed in the controller and the label switch according to network management services.

The remainder of this paper is organized as follows. In section 2, we described each network management service and its related managed objects in the GSMP interface. We discussed the locations of network management functions according to network management services in section 3. Finally, section 4 presented concluding remarks with some issues for further research.

\section{Managed Objects for Network Management Services}

\subsection{Approaches to Selection of Managed Objects}

There have been two studies of managed objects (MOs) in the GSMP interface: GSMP protocol MIB of RFC 3295 [5] and GSMP NM service MIB [6]. The managed objects of RFC 3295 are only defined to configure, monitor and maintain GSMP protocol entity. GSMP protocol MIB cannot support traditional network management services such as fault, configuration, accounting, performance, and security management services. GSMP NM service MIB includes managed objects to support network management services in the GSMP interface. Managed objects of this MIB are based on information elements of GSMP messages. GSMP NM service MIB 
is classified into the configuration group, the connection group and the performance group.

For NM services in the GSMP interface, there will be two approaches to choosing the relevant MIBs. The one approach is using the existing managed objects, which are defined in ATM-MIB [7] or MPLS-MIB [8]. In this approach, new managed objects will be defined if the existing managed objects could not be used for supporting a specific network management service. The advantage of this approach is that new definitions of managed objects will be minimized. The other approach is to use GSMP NM service MIB [6]. In this approach, network management functions will be simply mapped with the GSMP functions, because GSMP NM service MIB was defined to accommodate the information elements of GSMP messages.

\subsection{Configuration Management}

GSMP configuration messages permit the controller to discover the capabilities of the switch. Three configuration request messages have been defined in the GSMP: Switch, Port, and Service messages [3]. In the existing MIBs [7-8], there are no managed objects or tables, which are mapped with the GSMP configuration messages. To support switch and service configuration in the GSMP interface, it is required to define new configuration tables. Table 1 shows GSMP configuration messages and their related management tables in GSMP NM service MIB [6]. The columnar objects of these table entries are based on the information elements of the GSMP configuration messages.

Table 1. Configuration Tables of GSMP NM Service MIB

\begin{tabular}{ll}
\hline GSMP Messages & Configuration Tables \\
\hline Switch Configuration & gsmpSwitchConfTable \\
Port Configuration & gsmpInterfaceConfTable \\
& gsmpPortServiceMapTable \\
Service Configuration & gsmpServiceConfTable \\
\hline
\end{tabular}

The entry of interface configuration table represents the configuration information of a single switch port. The entries of service configuration table are the service lists of the switch, which are characterized by traffic and quality of service (QoS) parameters. The entries in the service mapping table of a port are service lists supported by the specific port. Figure 2 shows the relationship among interface configuration table, service configuration table and service mapping table of a port. 


\section{InterfaceConfIndex}

\begin{tabular}{|c|l|l|}
\hline $\mathbf{X}$ & & $\begin{array}{l}\text { One or } \\
\text { More }\end{array}$ \\
\cline { 1 - 2 } $\mathbf{Z}$ & & \\
\hline & \\
& \\
\cline { 1 - 1 } &
\end{tabular}

InterfaceConfTable

\begin{tabular}{|l|l|l|}
\hline$X$ & SID-1 & CSID-1 \\
\hline$X$ & SID-1 & CSID-2 \\
\hline$X$ & SID-2 & CSID-1 \\
\hline$Y$ & SID-1 & CSID-2 \\
\hline$Z$ & SID-1 & CSID-1 \\
\hline$Z$ & SID-2 & CSID-1 \\
\hline & & \\
\hline
\end{tabular}

PortServiceMapTable

SID : Service ID CSID : Capability Set ID

\begin{tabular}{|l|l|l|}
\hline SID-1 & CSID-1 & Traffic/QoS Para \\
\hline SID-1 & CSID-2 & Traffic/QoS Para \\
\hline SID-2 & CSID-1 & Traffic/QoS Para \\
\hline & & \\
& & \\
\hline
\end{tabular}

ServiceConfTable

Fig. 2. Relationship among Configuration Tables

\subsection{Connection Management}

For connection management service, we can use traffic parameter, in-segment, outsegment, and cross-connect tables of the existing MIB [7-8] or the GSMP service MIB [6]. Figure 3 shows the relationship among the interface configuration table and the connection related tables. A connection is modeled as a cross-connect consisting of in-segment and out-segment at a switch. These segments are related to input and output interfaces, and their characteristics are defined by the entries of the traffic parameter table. The interconnection of the in-segment and out-segment is accomplished by creating a cross-connect entry.

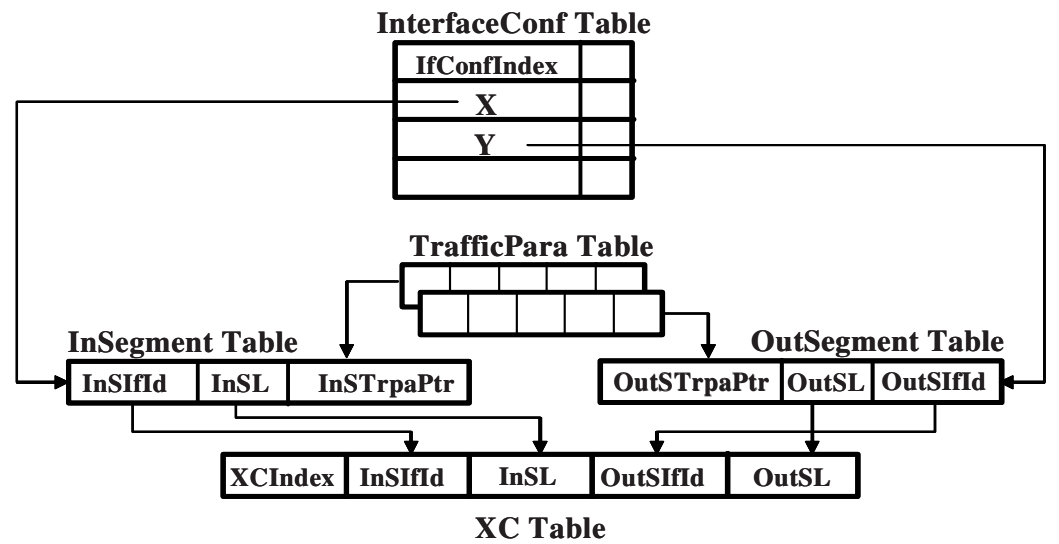

If : Interface, Conf : Configuration , S : Segment , L : Label , Id : Index, TrpaPtr : Traffic Parameter Row Pointer, XC : Cross-Connect

Fig. 3. Relationship among Connection Management Tables 


\subsection{Performance Management}

The performance messages of GSMP permit the controller to request the values of various hardware counters associated with the connections, input and output ports [3]. For connection performance management, we can use segment performance tables of the existing MIB or the GSMP NM Service MIB. Each entry of the interface performance table in the existing MIB or the GSMP NM Service MIB will be used for a port performance management. However, the columnar objects of existing table entries cannot be exactly mapped with the GSMP performance counters. The GSMP NM service MIB has interface and label performance tables, which are designed to be fully mapped with the GSMP performance messages.

\subsection{Event Management}

GSMP allows the switch to inform the controller of asynchronous events. The event messages defined in GSMP are Port Up, Port Down, Invalid Label, New Port, Dead Port and Adjacency Update messages [3]. GSMP protocol MIB [5] defines notifications, which are mapped with the GSMP event messages. Table 2 shows GSMP events and their related notifications in the GSMP protocol MIB.

Table 2. GSMP Event and Notification

\begin{tabular}{ll}
\hline Events & Notifications \\
\hline Port Up & gsmpPortUpEvent \\
Port Down & gsmpPortDownEvent \\
Invalid Label & gsmpInvalidLabelEvnet \\
New Port & gsmpNewPortEvent \\
Dead Port & gsmpDeadPortEvent \\
Adjacency Update & gsmpAdjacencyUpdateEvent \\
\hline
\end{tabular}

\section{Distribution of Network Management Functions}

Network management functions can be located either in the controller or the label switch, but the location has not been clearly defined for the GSMP interface. We will discuss the locations of NM functions according to network management services.

\subsection{NM Function for Connection Management}

Connections are usually classified into two types: a dynamic connection is established by the signaling protocol, and a provisioned connection is configured by the network management function. In the GSMP interface, switch resources for dynamic connections are managed by the controller, which admits a new dynamic connection, and commands the label switch to setup the admitted connection. The switch merely establishes the requested connection and responds to the controller [9]. 
Switch resources for provisioned connections will be managed by the controller or the label switch according to the location of connection management function. If the switch has connection management function, then it manages its resources for provisioned connections. This means that switch resources are managed by the controller or the label switch according to connection types. This separated management of switch resources will cause resource reallocation or inefficient resource usage. On the other hand, if the controller has connection management function, it will manage all switch resources for the dynamic and provisioned connection, so inefficient resource usage or resource reallocation problem can be dissolved [10].

Figure 4 shows the reservation and establishment procedures for a provisioned connection in the GSMP interface, where connection management function is located in the controller.

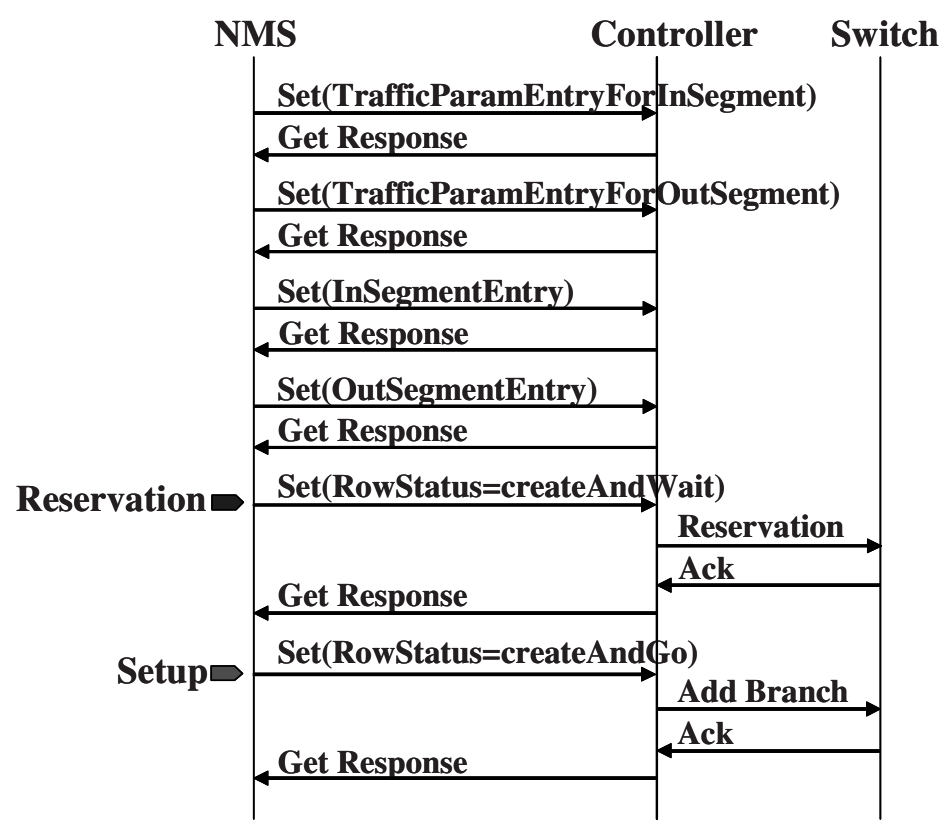

Fig. 4. Reservation and Establishment Procedures for Provisioned Connection

Network management station (NMS) first sets segment entries and their associated traffic parameter entries. After the segments are successfully created, the manager will request the controller to create a cross-connect entry. If the row status of the crossconnect entry is "createAndWait", then the controller commands the label switch to reserve the provisioned connection by sending GSMP Reservation message. After the controller receives the acknowledgement, it will complete the reservation of the provisioned connection by returning simple network management protocol (SNMP) Get Response message to the manager. 
NMS will activate the reserved connection by setting "createAndGo" in the row status of cross-connect entry. Then, the controller commands the label switch to establish the provisioned connection by sending GSMP Add Branch message. The manager deletes the provisioned connection by setting the row status of the cross-connect entry as destroy. Then, the controller requests the switch to delete the provisioned connection by sending GSMP Delete Tree message.

\subsection{NM Function for Performance Management}

Figure 5 shows information flows according to the locations of performance NM function.

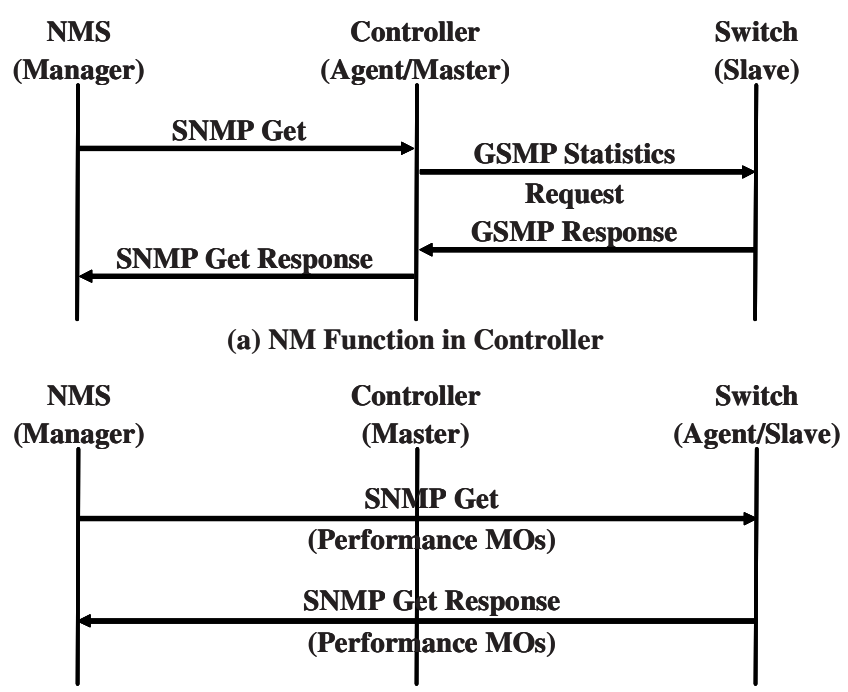

(b) NM Function in Switch

Fig. 5. Information Flows for Performance Management

If performance management function is located in the controller as shown in Fig. 5(a), the NMS issues SNMP Get message with performance related MOs to the controller. Then, the controller commands the label switch to collect performance information by sending GSMP Statistics message [3]. The controller returns SNMP Get Response message to the manager, when it receives the response from the switch. These information flows require mapping procedures between NM functions and GSMP functions, because NM functions are only in the controller.

If the switch has performance management function (see Fig. 5(b)), then the NMS could directly query the label switch about the performance information without mapping overhead in the controller. This direct interaction is feasible because performance information is actually collected and maintained by the switch. 


\subsection{NM Function for Fault Management}

Figure 6 shows information flows according to the locations of event NM function.

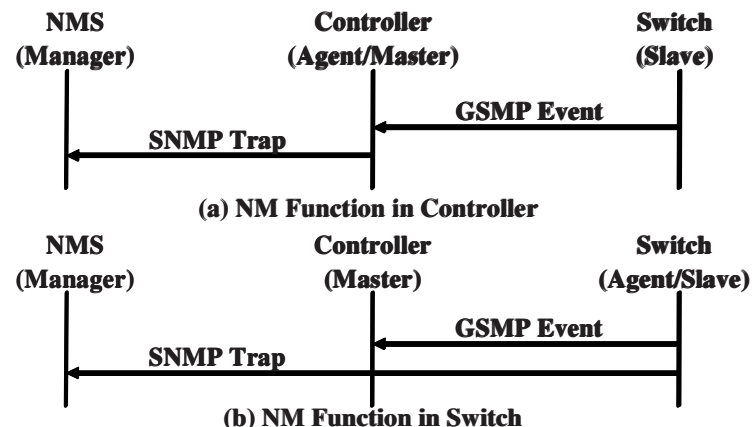

Fig. 6. Information Flows for Event Management

If fault management function is located in the controller as shown in Fig. 6(a), the controller maps the received GSMP event [3] into the SNMP Trap message to be transferred to the manager. This mapping overhead will cause a barrier for rapid fault management. If the switch has fault management function (see Fig. 6(b)), then the switch would inform the event to the controller and manager simultaneously. This direct notification is feasible, because event detection can be accomplished by the switch itself.

\subsection{Locations of NM Functions}

Figure 7 is our proposed NM model in the GSMP interface, which has distributed NM functions in the controller and the label switch according to NM services.

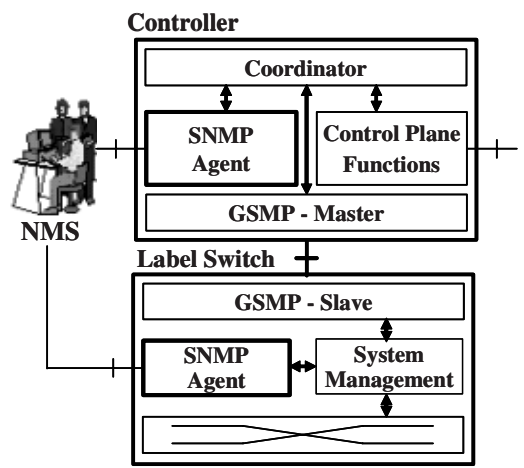

Fig. 7. Distributed NM Model in GSMP Interface 
In the GSMP interface, the controller implements control plane functions, which are supported by signaling and routing protocols [9]. This means that the controller completely makes decisions of connection and routing, and that the label switch merely responds to the commands from the controller. We put connection and configuration management functions in the controller, because connection admission control and service configuration are performed by the controller. On the other hand, we put NM functions for performance and fault management services in the switch, because the maintenance of performance information and event detection can be accomplished by the switch itself.

\section{Conclusion}

GSMP provides an open interface used to separate the data plane from the control plane. We guided the usage of MIB for each network management service in the GSMP interface. For GSMP connection and performance management functions, GSMP NM service MIB or existing MIB can be used. It is required to use configuration tables in GSMP NM service MIB, because existing MIB has no managed objects or tables, which can configure switch and service in the GSMP interface. Notifications in GSMP protocol MIB can be used for event management.

We proposed the network management model in the GSMP interface, which has distributed management functions in the controller and the label switch according to network management services. Our distributed NM model is based on which node controls connection resources, configures service and switch, collects performance data and detects events in the GSMP interface.

As the next step of our studies, we will extend and generalize the network management model of the GSMP interface and apply the generalized model to other open interfaces.

\section{Acknowledgement}

This work was supported in part by the Korea Science and Engineering Foundation (KOSEF) through OIRC project, and by a grant from 2004 Research Fund of Andong National University.

\section{References}

1. Nils Bjorkman et al., "The Movement from Monoliths to Component-Based Network Elements," IEEE Communications Magazine, January 2001.

2. Thomas M. Chen, "Evolution to the Programmable Internet," IEEE Communications Magazine, March 2000.

3. A. Doria, et al., "General Switch Management Protocol,” RFC 3292, June 2002.

4. YW Cha et al., "Network Management for GSMP Interface," Internet draft, <draft-chagsmp-management-01.txt>, November 2002. 
5. H. Sjostrand et al., "Definitions of Managed Objects for the General Switch Management Protocol," RFC 3295, June 2002.

6. YW Cha et al., "Definitions of Managed Objects for Network Management Services in General Switch Management Protocol (GSMP) Interface," Internet draft, <draft-cha-gsmpservice-mib-00.txt>, November 2002.

7. K. Tesink et al., "Definitions of Managed Objects for ATM Management," RFC 2515, February 1999.

8. Cheenu Srinivasan et al., "MPLS Label Switch Router Management Information Base Using SMIv2," Internet draft, <draft-ietf-mpls-lsr-mib-09.txt>, October 2002.

9. A. Doria, et al., "General Switch Management Protocol Applicability," RFC 3294, June 2002.

10. YW Cha et al., "Network Management Services in GSMP Open Interface," International Human.Society@Internet Conference, June 2003. 